REVIEW

\title{
THERAPEUTIC MOLECULES TARGETING THE MALIGNANT B CELL MICROENVIRONMENT OF CHRONIC LYMPHOCYTIC LEUKEMIA
}

\author{
Georgiana Ene ${ }^{1}$, Ana-Maria Vlădăreanu ${ }^{1}$, H. Bumbea ${ }^{1}$ \\ ${ }^{1}$ Hematology Clinic, University Emergency Hospital Bucharest
}

Corresponding author: Georgiana Ene

Email: georgi_dap@yahoo.com

\begin{abstract}
Although valuable advances have been made in the hematological field over the past years, most of the B-cell malignancies remain incurable, as malignant $B$ cells retain the ability to respond to a variety of microenvironment signals, providing additional opportunities in the development of therapeutic interactions. The role that the microenvironment has in the natural history of malignant B cell of chronic lymphocytic leukemia (CLL) appears to favor the development of new treatment modalities aimed at interrupting the interaction between malignant $B$ cells and microenvironment. At this moment, the therapeutic approaches whose target is the CLL microenvironment or the signaling pathways associated with CLL microenvironment are one of the most important therapeutic strategies. Interactions taking place within the tumor microenvironment are targeted by multiple clinical trials, and preliminary results are favorable. Chronic Lymphocytic Leukemia (CLL) is a size of complexity because leukemic cells are grown and protected by anti-cancer therapies by the components constituting the tumor microenvironment in lymphoid organs (e.g., endothelial cells, dendritic cells (CD), T cells, myeloid-derived suppressor cells (MDSCs), monocytederived nurse-like cells (NLC)). Current standard therapy in CLL combines chemotherapy with an anti-CD20 monoclonal antibody. This combination induces substantial toxicity and is not curative, as most patients relapse. Recent advances using kinase inhibitors, such as ibrutinib and idelalisib or BCL2 signaling inhibitors e.g. Venetoclax, indicate a major change promising to treat chronic lymphocytic leukemia without chemotherapy. At present, these therapeutic agents do not provide complete responses and should be administered continuously by the patient in order to avoid recurrence/relapse of the disease. Resistance to ibrutinib has already been detected in patients with high genetic risk. This problem requires the identification of therapies that combine agents with distinct mechanisms of action.
\end{abstract}

KEYWORDS: chronic lymphocytic leukemia, microenvironment, malignant B cell, treatment

\section{INTRODUCTION}

At the current moment the standard of treatment for young patients with CLL is still chemotherapy, the most commonly used scheme being R-FC (rituximab, fludarabine and cyclophosphamide). However, chemotherapy results are often unfavorable, particularly in 
some subgroups of patients, for instance with increased cytogenetic risk (e.g. 17p deletion) or in elderly patients (over 70 years) with low tolerability to treatment.

Lower scores due to chemotherapy have favored the introduction of new therapeutic agents (e.g. ofatumumab, bendamustine, alemtuzumab), and have led to the development of alternative therapeutic regimens targeting interactions between CLL cells and microenvironment (e.g. lenalidomide, chemokine receptors and BCR or BCL2 signaling inhibitors) [3]. When engaged in adherence to stromal cells, CLL cells are resistant to the cytotoxic effects of drugs commonly used in the treatment of patients with CLL, for example corticosteroids and fludarabine [4]. This adhesion-mediated drug resistance mechanism may be an explanation for the relapse of the disease and the presence of residual minimal disease (MRD) in bone marrow of patients with CLL $[4,5]$.

The CLL microenvironment has gained a lot of attention in recent years, due to the introduction into the therapeutic regimens of small molecule inhibitors targeting intermicroenvironment [6]. These investigators target BCR associated kinases (the most obvious examples being ibrutinib and idelalisib) and BCL2 (venetoclax) have changed the fate of patients with CLL in terms of survival and quality of life, as they have led to sustained remissions in refractory patients or disease in progression, including those who show genetic changes with unfavorable prognosis (e.g., del $17 p$ or del 11q).

\section{THE ROLE OF TUMOR MICROENVIROMNENT IN CURRENT THERAPEUTIC STRATEGIES}

Hematological cancers arise from the specialized microenvironments and appropriate their development, such as bone marrow (B.M) and secondary lymphoid organs. Both B.M and secondary lymphoid organs are distinct microenvironments, each regulate different stages of maturation and differentiation of lymphocytes. At the B.M level, hematopoietic stem cells and mature B cells develop from common progenitors. Mature B cells migrate into the secondary lymphoid organs where they are exposed to antigen (Ag) in germinal centers of secondary lymphoid follicles [7].

The tumor microenvironment is made up of a variety of factors, such as auxiliary stromal cells, $\mathrm{T}$ cells that promote tumor expansion and its resistance to treatment and malignant blood cells that are in a relationship of interdependence with the signals from the microenvironment. Another major factor in the microenvironment of $\mathrm{B}$ cell lymphomas is angiogenesis that acts by at least two mechanisms: autocrine stimulation and paracrine. Another aspect is the mechanisms of immune escape of malignant $B$ cells that includes altered expression of surface molecules involved in recognition of immune cells, defects in formation of immunological synapses and coopting the network of immune control points by the tumor microenvironment [8].

Targeting specific intracellular pathways involved in interactions with CLL cells / tumor microenvironment may represent a new and attractive option to increase the therapeutic weapons used in CLL [9]. An overview of medicines which can be or are used to target specific molecules involved in interactions between CLL cells and the tumor microenvironment is proposed in this article.

However, the immune and stromal cells of the tumor microenvironment (TM) are genetically stable compared to neoplastic cells, thus being less susceptible to classical therapeutic resistance mechanisms, and targeting TM has become a mandatory therapeutic strategy. Due to the paradoxical capacity of TM to promote and block tumor growth, the therapeutic approach explored was the development of treatment strategies that restore the TM balance rather than simply destroy its components [8]. A revolutionary breakthrough in cancer therapy has been the use of immunotherapy, which targets the evasion mechanisms of T-cell by tumors [1]. In addition, studying molecular bases which mediates the interactions of CLL cells with the various components of TM may be useful in understanding mechanisms of neoplastic development and progression and identification therapeutic targets to be achieved with the help of specific drugs in combination with conventional chemical immunotherapeutic agents in selected CLL subsets [9]. 
Another important aspect is that microenvironmental niches may provide a safe haven from cytotoxic drugs, favoring relapse. Therefore, it is crucial to identify molecular interactions that bind malignant $\mathrm{B}$ cells to the microenvironment, and it is also important to investigate whether neoplastic clone responses to microenvironmental signals define abnormalities associated with malignancy compared to normal B cells.

Although at first, approaches to modulate microenvironmental elements and their orientation to CLL cells may have therapeutic potential, the condition is that we can exploit and fully understand the complex interactions that occur in vivo [10]. The interactive dynamic established between CLL cells and the microenvironment apparently promotes the growth and survival of CLL cells in vivo. Targeting this dynamics is therefore an attractive way for therapy, the goal being to induce apoptosis of CLL cells and / or to increase the sensitivity of CLL cells to current therapies [11]. These approaches could be divided into two basic categories, namely:

1. strategies that block the interaction of survival / growth factors with receptors

2. strategies that disrupt signaling pathways in CLL cells that are activated by survival / growth factors [11]

CXCR4 antagonists are included in the first category because they block the CXCL12 interaction with its receptor. AMD3100 (Plerixafor) or T140 may diminish the survival support provided by the CXCR4 to microenvironment and may increase the sensitivity of CLL cells to the cytotoxic effects of leukemia drugs such as fludarabine monophosphate $[11,12]$. The second category includes components that target the survival or growth pathways of leukemic cells promoted by microenvironmental factors. This category includes inhibitors of:

- phosphatidylinositol-3-kinase (CAL-101)

- spleen tyrosine kinase (SYK)(fostamatinib disodium/R406)

- tyrosine kinase Bruton (BTK)

(PCYC-1103-CA)

- BCL-2 (venetoclax, obatoclax/ GX15070 and ABT-263) [11]

\section{SIGNALING PATHWAYS ASSOCIATED WITH CLL MICROENVIRONMENT THAT ARE FREQUENTLY TARGETED BY THERAPEUTIC STRATEGIES}

\section{$B C R$ signaling pathway}

In the last decade, studies on the structure and function of the $\mathrm{B}$ cell antigen receptor $(\mathrm{BCR})$ have redesigned the nature of the chronic lymphocytic leukemia [13]. BCR signaling pathway is important in the pathogenesis of CLL and there is evidence to support this, but the precise mechanism that triggers the activation of $\mathrm{BCR}$ remains controversial. BCR activation in MT has emerged as a central oncogenic pathway, essential for the survival and proliferation of CLL [14]. BCR and MT tissue signals converge on several intracellular signaling pathways, including the PI3K-AKT axis [14].

The BCR stimulation signal plays a significant role in the development and prognosis of CLL as disease progression is closely linked to variable BCR area mutations and CLL cells express the IGHV sequence restrictively and no mutation of Ig and / or ZAP$70+$ has a priority response to $\mathrm{BCR}$ stimulation [4].

BCR pathway is an engine of CLL progression and BCR stimulation signals plays a significant role in the development and prognosis of CLL, so targeting BCR signaling pathway is an important therapeutic strategy.

It has been demonstrated that inhibitors of BCR signaling pathways (Figure 1) have low molecular weight, show a great clinical activity and target BTK kinases, PI3K and SYK.[15] $\mathrm{BTK}$ is a tyrosine kinase non-receptor member of the Tec family kinases, which is activated by LYN and SYK kinases, which play a role in the proliferation and differentiation of $B$ cells and which lead to activation of the NF- $\mathrm{KB}$ signaling pathway and protein kinase activated by mitogen (MAPK) [3]. By inhibiting Bruton tyrosine kinase (BTK), which is an essential molecule in BCR signaling transduction, inhibit survival and proliferation depend on CLL microenvironment, including those that feature BCR and CD40 [16]. By inhibiting SYK and PI3K, the interaction between CLL cells and the microenvironment is blocked, and pro-apoptotic signals are promoted by inhibiting BTK, PI3K 
and SYK [4]. Inhibitors of SYK (fostamatinib disodium), BTK (ibrutinib) and PI3K (GS1101) have common characteristics in the treatment of chronic lymphocytic leukemia, due to the fact that these drugs can cause a transient increase in lymphocytes during the first week of treatment due to the mobilization of CLL cells in the peripheral blood from tissue, lymph nodes act on reducing their size, and bone marrow suppression is reasonable $[4,17$ to 19$]$.

Ibrutinib is an oral inhibitor that blocks the phosphorylation and activity of BTK kinase, which was approved in 2014 for the treatment of mantle lymphoma and chronic lymphocytic leukemia. Ibrutinib block B cell receptor in a number of microenvironments: lymph nodes, bone marrow and peripheral blood. Early lymphocytosis and gradual reduction of organomegaly followed by lymphocyte normalization are typical effects of ibrutinib therapy, linked to inhibition of CLL cell proliferation and induction of cell death in vivo. Changes that occur in the tumor microenvironment of chronic lymphocytic leukemia include:

i. blocks BTK kinase activity

ii. blocks pro-survival signals derived from CLL microenvironment in vitro, such as those arising from contact with nurse-like cells and CD40 ligand, BAFF, fibronectin (FN), IL-6, IL-4, TNF $\alpha$

iii. inhibits CLL cell proliferation

iv. inhibits chemotaxis toward CXCL12 and CXCL13

v. inhibits integrin-mediated adhesion

vi. inhibits the release of CCL3 and CCL4 chemokines in vitro [6]

It also induces sustained remission in patients with refractory / recurrent disease, including in patients with high-risk genetic lesions (e.g. del17p and del11q), as well as in previously untreated elderly patients ( $>65$ years).[6] Ibrutinib is currently being explored in various combinations of chemotherapy or associated with monoclonal antibodies in order to increase the overall remission rate. In theory, Ibrutinib can reduce the risk of new molecular aberrations and clonal evolution [20]

The PI3K / AKT signaling pathway

It has a central role in regulating cellular events that support the survival of B CLL cells.
PI3K regulates several cellular functions including survival, migration and cell growth following BCR activation, chemokine receptor and activation of integrin signaling. The predominant form expressed by hematopoietic cells is $\mathrm{PI} 3 \mathrm{~K} \delta$, which plays a critical role in homeostasis and B cell function [3].

Idelalisib (encoded GS-1101 or CAL101 ) is a specific inhibitor of $\mathrm{PI} 3 \mathrm{~K} \delta$, which promotes apoptosis, CLL cell migration. Idelalisib was also approved in 2014 for treatment of patients with CLL who had previously been treated and can be used as monotherapy or in combination with rituximab and / or bendamustine. Idelalisib induces the same as ibrutinib early lymphocytosis followed by normalization of lymphocyte count. His action on CLL microenvironment consists of:

- antagonizing survival signals such as contact with nurse-like cells and CD40 ligand

- stimulates BCR, TNF $\alpha$, fibronectin

- reduces CLL cell chemotaxis in order to release CXCL12 and CXCL13, CCL3 and CCL4 in vitro by CLL cells

- reduction of CLL adhesion to VCAM-1 (vascular cell adhesion molecule-1) and fibronectin [6]

OSU-T315 is a novel PI3K / AKT pathway inhibitor with a unique mechanism that directly cancels AKT signaling and induces apoptosis in CLL cells by suppressing AKTmediated activation by the $\mathrm{BCR}$ receptor, CD49d, CD40 and Toll-like receptor [21]. Liu et al conducted a study which compared the OSU-T315I activity in CLL-derived cell lines and in primary CLL cells operating in normal lymphocytes [21]. Although Ibrutinib and Idelalisib show a clinically remarkable response in CLL patients, recent studies have identified refractory patients on these agents such as Ibrutinib by developing a BTK mutation (BTKC481S).Therefore, OSU-T315 may prove to be very useful in the treatment of patients with high risk CLL, such as del $17 p$, non-mutant IGVH status or those who develop resistance to Ibrutinib [21].

Other therapeutic goals targeted are SYK kinases, adhesion molecules VLA-445 or CD44. SYK that belongs to the non-receptor kinase family SYK / ZAP70 is a key component of BCR signaling with a role in activating it. 
SYK signaling is required for B cell growth, their proliferation, and survival. Clinical trials conducted on murine models have shown that mice deficient in SYK has a prohibition regarding the transition from pro-B to pre-B stage [4,22]. Fostamatinib (FosD, R788) is an oral inhibitor available for SYK, which induced partial responses in patients with refractory CLL, but the drug is still in the test phase.[6] R406 (the active metabolite of Fostamatinib) is a competitive ATP kinase inhibitor a key role in signaling $\mathrm{Fc}$ receptor activators and $\mathrm{B}$ cell receptor. R406 demonstrates its efficacy in CLL and other $\mathrm{B}$ cell malignancies by disrupting BCR signals and interactions with microenvironment $[4,23]$. Studies conducted by Herman et al. [24] concluded that the expression of $\mathrm{NfkB}$ and MYC genes decreases under the action of fostamatinib [4,24]. Expression of the markers CD69, CD86 and the percentage of Ki67-expressing CLL cells are reduced by the action of fostamatinib [4]. The cytotoxic effects of the SYK inhibitor are associated with SYK protein expression and are more pronounced in cases of CLL ZAP70+ or with non-mutant IGVH status [4].

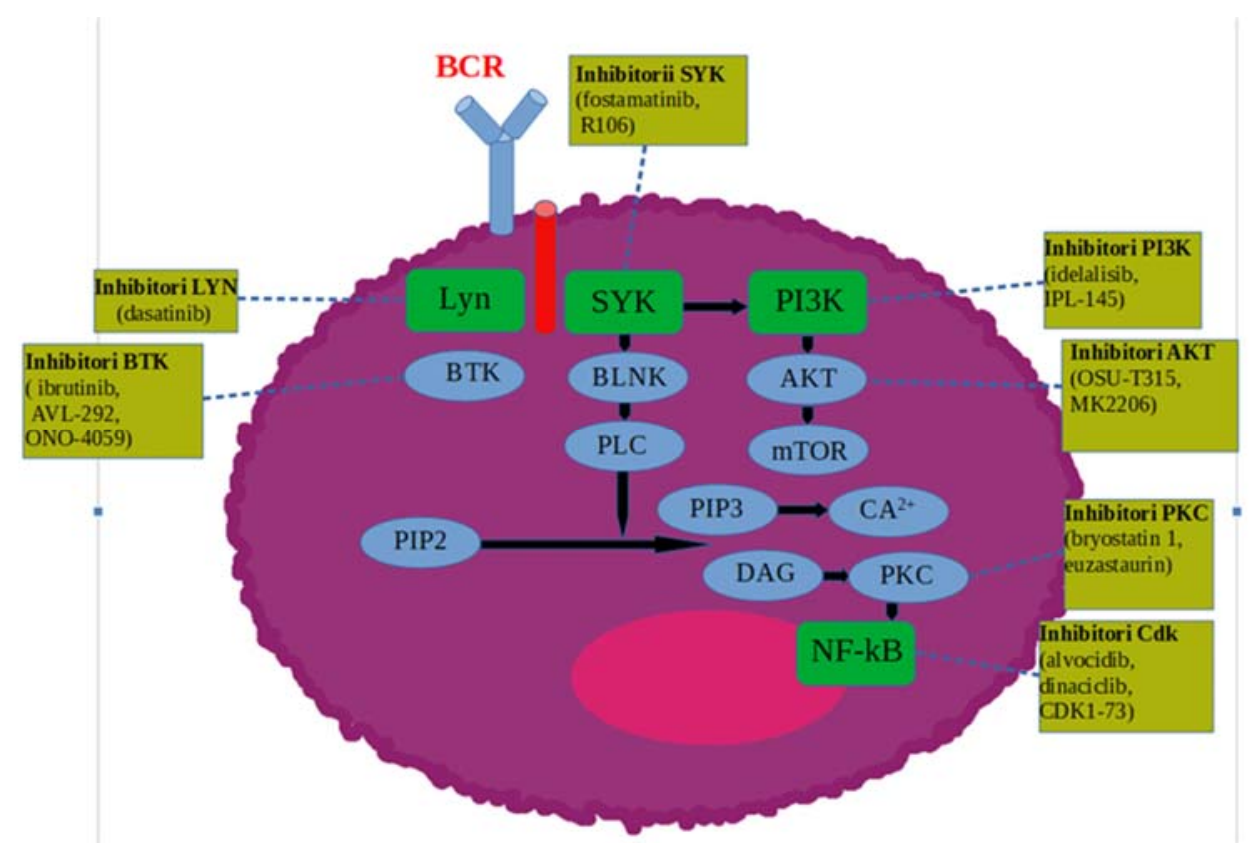

Figure 1 - The image sketches the kinase inhibitors that are already approved or with therapeutic potential in CLL, the BCR signaling pathway and their biological targets [21].

Abbreviations: $\mathrm{BLNK}=\mathrm{B}$ cell linker; $\mathrm{CDK}=\mathrm{Cyclin}-\mathrm{dependent}$ kinase; $\mathrm{DAG}=$ diacilglycerol; Lyn=a member of the Src family of tyrosine kinase proteins; NF-kB=nuclear factor $\mathrm{kB}$; PIP2=phosphatidylinositol 4,5bisphosphate; PIP3= phosphatidylinositol $(3,4,5)$-trisphosphate; $\mathrm{PKC}=$ protein kinase $\mathrm{C}$; $\mathrm{PLC}=$ phospholipase $\mathrm{C} ; \mathrm{mTOR}=$ the mammalian target of rapamycin .

\section{Signaling axis CXCR4 / CXCL12}

This axis represents a therapeutic target in CLL more intensively studied. The therapeutic target of CXCR4 inhibits CLL cell migration, making the malignant cell more susceptible to drug-induced apoptosis in peripheral blood.[16] CXCR4 antagonists have been developed which include peptide CXCR4 antagonists (BKT140), small molecule CXCR4 antagonist (AMD3100, now called plerixafor) and CXCR4 antibodies (MDX1338/93656BMS).[6,25] CXCR4 antagonists inhibit CXCL12-mediated signaling, chemotaxis and drug resistance induced by stromal cells, and they can also block the CLLstromal interactions after which they can mobilize CLL cells from their blood-protecting microenvironments, making them accessible to conventional drugs [4].

In CLL, plerixafor not only inhibits the activation of signaling mediated by CXCL12 but also disturbs interactions between CLL and nurse-like cells as well as CLL interactions and stromal bone marrow cells, blocks survival stimuli and interferes with the survival signal provided by CXCL12 [6,26]. In chronic 
lymphocytic leukemia, mobilization and sensitization of leukemic cells could be achieved by combining a CXCR4 antagonist with conventional cytotoxic agents (fludarabine, cyclophosphamide), monoclonal antibodies (rituximab, alemtuzumab) or immune chemotherapy [4]. Andritsos et al. [27] conducted a study on the association between AMD3100 and Rituximab, and the results were promising. From the data obtained, it seems that this combination could represent a new therapeutic pathway for patients with relapsed CLL $[4,27]$.

\section{DRUGS THAT TARGET THE CLL MICROENVIRONMENT}

The immunosuppressive nature of the MT proves to be a major barrier for the treatment of malignant $\mathrm{B}$ cell diseases. For example, monotherapy with drugs that target molecules in the immune blockade control, such as PD-1 (pidilizumab), had promising results in a subset of patients and highlighted tumor immune evasion [8,28]. Therefore, in order to combat immune evasion, it will most likely be necessary to use combinations of drugs with multiple therapeutic targets that have potential synergistic anti-tumor effects. A notable preclinical success was obtained when the PDL1 antibody was combined with the kinase inhibitor (Ibrutinib) [29]. This combination showed synergistic activity in preclinical models of lymphoma, with a cure rate of about $50 \%$ and delayed tumor growth in mice which were previously insensitive to treatment with Ibrutinib [8]. Ibrutinib targets both CLL cells by inhibiting BCR signaling and the survival of Th2-type $\mathrm{T}$ cells by inhibiting IL-2 inducible cellular $\mathrm{T}$ cell kinase, a vital protein in the survival of Th2-type T cells [30].

Studies have recently found that immunomodulatory drugs (IMiDs), such as thalidomide, lenalidomide and pomalidomide, bind a target protein called cereblon (CRBN). The ability of immunomodulators to target cereblon and the ubiquitin proteasome system modulates the expression of critical transcription factors that co-stimulate $\mathrm{T}$ cells, simultaneously degrading B cell function [8]. In addition, IMiDs stimulates the expansion and activation of $\mathrm{CD} 8+\mathrm{T}$ cells while reducing the activity of cytokines derived from CD4+ T cells, promotes differentiation of Th1-type $\mathrm{T}$ cells and polarization of Th2-type $\mathrm{T}$ cells into a Th1 phenotype (IFN- $\gamma$, TNF- $\alpha$ ) [2,31]. Furthermore, inhibition of CXCL12 by IMiDs may interfere with the CXCR4-CXCL12 axis in the tumor microenvironment of chronic lymphocytic leukemia [2].

Lenalidomide has several mechanisms of action that can be simplified by organizing them as mechanisms of action in vitro and in vivo [32]. In vitro, lenalidomide has three main activities: direct anti-tumor effect, inhibition of angiogenesis and immunomodulation. In vivo, lenalidomide acts directly by inducing apoptosis of tumor cells, but also has actions with indirect effect such as: inhibition of osteoclast formation, inhibition of bone marrow stromal cell support, anti-angiogenic effects and immunomodulatory activity. Lenalidomide interfere with several components of CLL microenvironment and has a wide range of activities that can be exploited, for example:

- increases the proliferation and activity of the antigen mediated by the CD4 $+\mathrm{T}$ cell $[6,31,33]$

- enhances the antitumoral immune response mediated by NK (natural killer) cells and CD4+ T cells [6,34]

- restores the functional formation of immune synapses between $\mathrm{T}$ and CLL cells $[6,35]$

- regulates the PD-1 / PD-L1 immunosuppressive axis $[6,35]$

- improves T cell motility $[6,36]$

- activates B cells [6,37]

- interferes with the activity and proliferation of T-regulatory cells $[6,38]$ Due to the multiple mechanisms of action Lenalidomide can be used as monotherapy in patients with chronic lymphocytic leukemia refractory or relapsing, in combination with new therapies Rituximab or Ibrutinib or may be administered as initial therapy in elderly patients with CLL who cannot tolerate regimens more aggressive [6].

Lenalidomide is an oral immunomodulatory drug that repairs T-cell antitumor function and is efficacious in CLL clinical trials, particularly in patients with recurrent chronic lymphocytic leukemia [1]. It has been demonstrated that lenalidomide 
treatment of patients with CLL reduces the proportion of regulatory $\mathrm{T}$ cells $(\mathrm{CD} 4+\mathrm{CD} 25 \mathrm{hi}$ FOXP3 + ) and increases the proportion of $\mathrm{T}$ helper cells (Th17). In addition, a change in $\mathrm{CD} 8+\mathrm{T}$ cell activation status, namely the transition, from a proliferative phenotype to a cytotoxic one, was reported [11,39]. Lenalidomide also inhibits the bi-directional pro-survival course between endothelial cells and tumor cells, including pro-angiogenic signals [40]. In vitro, Lenalidomide is not directly cytotoxic for CLL cells, but changes the CLL-MT pro-tumor signals. Direct anti-CLL effects include inhibition of MT-induced proliferation. In CLL, combining lenalidomide with anti-PD-L1 can improve T-cell anti-tumor immunity because both agents block immunosuppressive signaling [41]. Also, Ibrutinib through ITK-mediated inhibition of Th2-type CD4 + T cells may have the additional immunomodulatory potential of IMiDs improving the immunity of Th1 type CD4+ T cells and CD8+. However, such clinical trials following the combination of lenalidomide / IMiDs with ibrutinib or other novel agents, are of great interest and need careful monitoring of the potential reactions [8]. Studies provides evidence supporting lenalidomide combination with PI3K inhibitors, and other potential inhibitors of BCR, and this is seen as a new chemo-immunotherapy in the future [4].

The combination of conventional agents such as IMiDs with immunotherapy or blocking immune checkpoints, should contribute to an immune tumor microenvironment to subsequent clinical benefits for patients. An example used in targeting tumor associated macrophages in Bcell neoplasms is illustrated by anti-CD47 antibodies. Macrophages are essential in many therapies. Phagocytic cells represented by macrophages and dendritic cells express SIRP $\alpha$ that binds to CD47. SIRP $\alpha$ CD47-mediated activation initiates a cascade of signal transduction pathways that result in the inhibition of phagocytosis [8]. This immune escape mechanism can be therapeutically targeted in hematological neoplasms with an anti-CD47 antibody that restores phagocytosis and eliminates tumor cells $[8,42,43]$.

Immunotherapies that block immune checkpoints and IMiDs offered CLL patients new therapies with sustained disease control, but for the benefit of these patients is essential that research efforts should continue and be extended to find a therapeutic combination that release immune antitumor responses. Also, regulation of tumor microenvironment pathways will need to be targeted by combined therapies to provide survival benefits for as many patients as possible [8].

The widespread availability of BCR signaling antagonists has altered the nature of CLL therapy. CLL control in the era of chemoimmunotherapy was strongly correlated with the quality and depth of remission. Failure to achieve at least a partial response after treatment with R-FC has a disastrous prognosis among patients, particularly those who have a high genetic risk [44]. On the other hand, the majority of patients treated with BCR signaling antagonists, eventually reaching durable remissions, but no better than a partial response, and achieving a complete response was rarely observed following monotherapy.

Idelalisib described above is a selective oral inhibitor for $\mathrm{PI} 3 \mathrm{~K} \delta$, and in terms of preclinical activity, it has been demonstrated that this molecule inhibits both intrinsic and external survival signals, including those generated by BCR signaling in CLL [2]. Inhibition of PI3K interfere with the retention of CLL cells in lymphoid tissues, reducing the ability of CLL cells to remain sequestered in the microenvironment. Another PI3K-targeted agent still in clinical development is IPI-145.

The first BTK inhibitor that came into clinical experience was Ibrutinib. The preclinical experience with Ibrutinib activity in CLL has demonstrated inhibition of both intrinsic survival and BTK-mediated survival signals [2]. In addition, since treatment with Ibrutinib results in the migration of CLL cells from the lymph node, inhibition of the chemokine that attracts CLL cells, could avoid the reformation or formation of a tumor-friendly micromedium and would increase the effectiveness of cytotoxic therapies [45].

Sorafenib is another promising therapeutic agent for chronic lymphocytic leukemia. In CLL cells ZAP-70+ sorafenib blocks CXCL12-induced phosphorylation of ERK and MEK pathways. ZAP-70+ CLL cells are more sensitive to the cytotoxic effects of sorafenib compared to ZAP-70- CLL cells 
$[4,46]$. The strength of this agent is to overcome the protective barrier of the CLL micromedium, in terms of chemokine signaling and prosurvival signals [4].

Dasatinib was recently approved for use in CLL and is a new chemotherapeutic agent without a protective microenvironment. Due to the fact that dasatinib inhibits all Src family tyrosine kinases, research has shown that dasatinib can inhibit BCR signal transduction and, additionally, block BCR-mediated survival of CLL cells [47]. Also, dasatinib interferes with the migration of CLL cells to CXCL12 by inhibiting the CXCR4 signaling pathway [4]. Amrein et al. [48] conducted a phase II study of dasatinib in relapsed and refractory CLL, and from the data obtained it was found that dasatinib exhibited intense activity in patients diagnosed with high risk CLL presenting del (11q) and the same time toxicity of myelosuppression is common [4,48]. Dasatinib has been used as monotherapy, combination with other drugs has not been studied.

Molecular pathways involving molecular structures associated with a poor prognosis in CLL and which are activated by CLL / micromedium interactions may be targeted either by CLL or blocking receptors specifically expressed by the microenvironmental cell populations, which in turn interact with CLL cells [9]. In this regard, a selective inhibitor of SYK R788 (Fostamatinib) is able to block BCR signaling in vivo, resulting in reduced proliferation and survival of malignant B cells $[9,49,50]$. Treatment with R406, the active form of R788, abrogates the pro-survival effects promoted by IgM stimulation and nurse-like cell co-culture in vitro $[9,49,50]$. Clinical trials have shown significant activity of R788 in CLL and non-Hodgkin's lymphoma [9,51].

Similarly, it has been demonstrated that selective inhibitor PI3K-CAL101 promote apoptosis in CLL primary cells ex vivo by interfering with signaling from the outsideinside BCR-mediated triggering [9,52,53]. Also have been reported to have therapeutic role molecules that interact with TLR7 and / or TLR9, that enter the BCR using cell and induce a long-term apoptotic cascade in CLL $[9,54]$. In addition, other drugs that use the BCR pathway by inhibiting Hsp90 protein (e.g. geldanamycin) may play a role in ZAP-70+ CLL cells, given the Hsp90 activity in ZAP-70 stabilization [9,55].

In addition to the molecular pathway associated with the BCR signaling cascade, other therapeutic agents that act by blocking specific receptors involved in interactions between CLL cells and the micromedium may be:

a) anti-CD38 antibodies (e.g. Daratumumab and SAR650984) which have been tested in multiple myeloma $[9,56]$

b) anti-CD49d antibody, Natalizumab

c) CXCR4 antagonists (e.g. T140 analogs, plerixafor (AMD100)) that can destroy the adhesive relationship between the stromal cells and CLL and can mobilize CLL cells from their protective microenvironments [5,9,57]

Clinical trials have evaluated the use of plerixafor in combination with rituximab in the treatment of patients with recurrent / refractory CLL [11,27]. Preliminary data showed that treatment with plerixafor induced mobilization of CLL cells in the blood, dose-dependent and that this is a promising approach regarding the release of CLL cells from the microenvironment [11]. Similarly, a soluble form of TACI, called Atacicept, was developed as a bait receptor to neutralize APRIL and BAFF [11]. In a Phase $1 b$ study involving patients with refractory and / or recurrent CLL, Atacicept promoted disease stabilization, which suggested that it could interfere with the proliferation or survival of CLL cells in vivo [11,58].

Venetoclax is the first drug approved that targets the BCL2 protein. BCL2 overexpression in CLL is associated with drug resistance and tumor cell survival. Venetoclax was effective among patients with poor prognosis, especially those whose tumors do not respond to fludarabine.

Obatoclax or GX15-070 is a pan-BCL2 inhibitor that has been shown in vitro to induce apoptosis of CLL cells and, in vivo a phase I study was conducted in patients with advanced CLL, which revealed that Obatoclax showed modest clinical and biological activity suggesting its use in a combination therapy approach [11,59-60]. Navitoclax (formerly ABT-263) is an experimental oral drug that has a similar activity as Obatoclax, except that do 
not target only BCL-2, but also BCL-XL and BCL-x [11,61]. In vitro, Navitoclax has been shown to rapidly induce apoptosis in various cell lines as well as in primary CLL cells, and a phase I clinical trial reported that this compound is safe and active in patients with relapsed CLL [11,61-63].

Another topical therapeutic strategy is targeting receptors expressed by normal microenvironmental cells or soluble factors that transmit signals in the combination of CLL cells and neighboring cells [9]. In this regard, the UCB35625 molecule, as selective CCR1 or anti CCR5 antibody PRO140 may be relevant given their role in CLL cell interactions with CD68+ macrophages or $\mathrm{T}$ cells [9,64-67]. Also, other drugs that can acquire interest in the treatment of chronic lymphocytic leukemia, could be antiTNF antibodies, such as adalimumab or certolizumab $[9,68-70]$.
Regarding CLL, some data suggest that Survivin (BIRC5) would be a potentially useful target for immunotherapy and for drugs capable of interfering with critical molecules [10,71].

Therefore, new strategies that disrupt interactions between CLL cells and cells in the tumor microenvironment are in constant evaluation. In addition, they are recognized as a major contributor to pathogenesis as well as a potential objective in the development of new therapies. So they can prove their effectiveness alone or used in combination with standard therapies that improve the outcome of CLL patients. In conclusion, future perspectives on the dynamics of cellular interactions and the effects of existing therapies on these dynamics would help substantially in developing optimal treatment strategies [45]. A schematic of all the alleged interactions and the drugs described in this paper is shown in Figure 2.

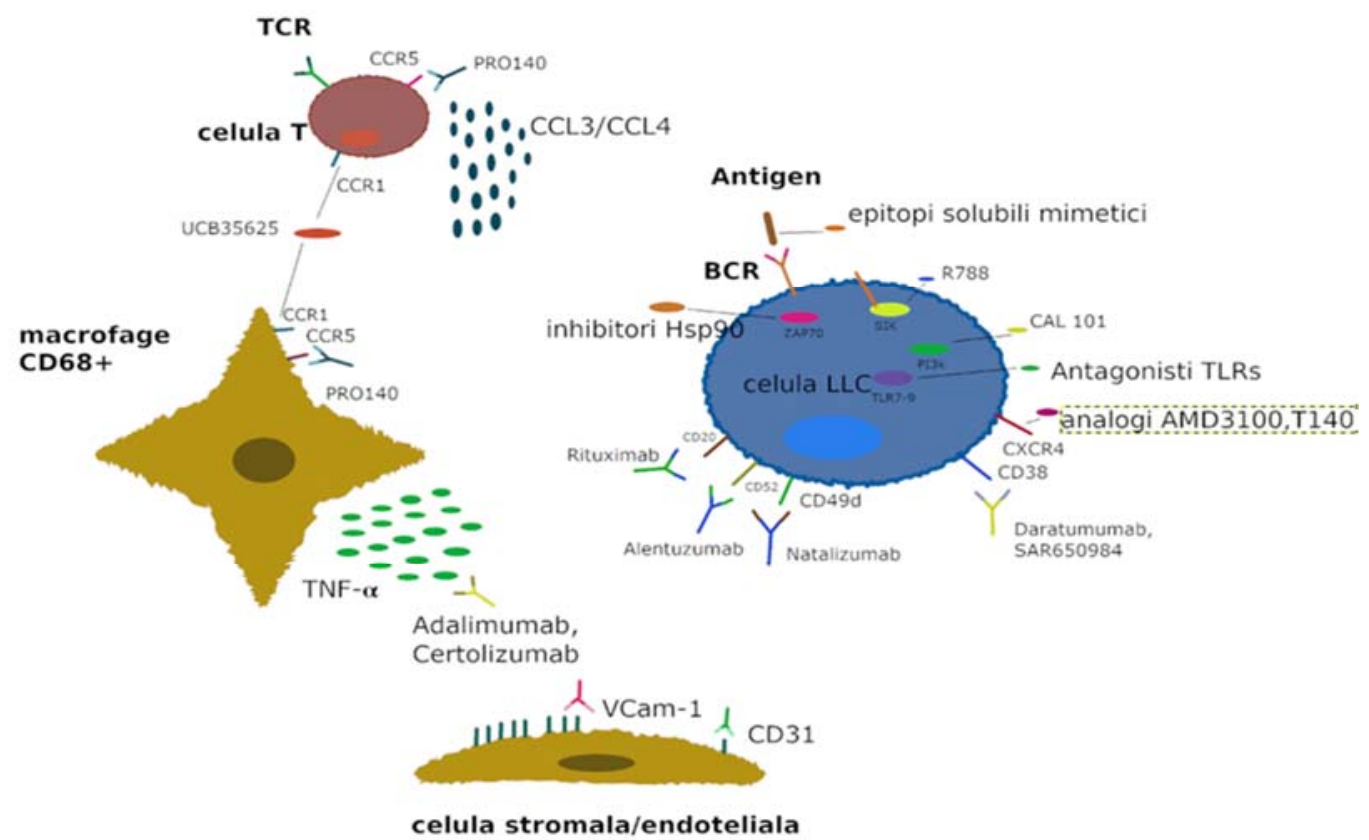

Figure 2 - A schematic of the interactions taking place in the microenvironment between CLL cells, CD68 + macrophages, $T$ cells and stromal/endothelial cells are described in the present paper together with available drugs and antibodies that block specific receptor / ligands pairs [9].

\section{CONCLUSIONS}

Currently still being tested and compared new drugs. The focus is on optimizing treatment strategies (using them as monotherapy or in combination with standard chemotherapy regimens or monoclonal antibodies), drug resistance mechanisms, and defining treatment failure.
At this point $\mathrm{BCR}$ associated kinase inhibitors (SYK, BTK, PI3K $\delta$ ) are the most advanced therapeutic solutions aimed CLL microenvironment and showing the highest clinical activity in CLL. The current enthusiasm for these new agents is justified by clinical activity and the lack of major side effects (e.g. myelosuppression), but the precise mechanism of action, the potential benefit of combinations 
with conventional agents, the usefulness of biomarkers such as CCL3, the durability of responses and the potential mechanism resistance must be explored [7].

New information about these drugs supports their application as monotherapy, but also for their use as a combination therapy (their association with rituximab, bendamustine, ofatumumab) using the dependence of CLL microenvironment. Potential mechanisms of resistance to BCR inhibitory drugs are not yet known, but causes are investigated and analyzed, and their discovery will become an innovative path in the future [4].

The therapeutic agents described above provide a broad basis for developing additional strategies to improve treatment efficacy in patients with CLL, especially in those at high risk. The development of these specific treatments, whether it be a single agent or in combination with conventional therapies, should improve the quality of life of patients with CLL. Close monitoring of the duration of the response to treatment, the risk of disease progression, secondary effects and minimal residual disease is also required [4].

In the following years, a stronger emphasis will be placed on those mysteries that are not fully understood as well as on the CLL microenvironment, which will most likely lead to a paradigm shift towards kinase inhibitor treatment in CLL which will set a new standard in patient care with CLL and which will benefit in the near future a large number of patients with chronic lymphocytic leukemia.

\section{REFERENCES}

[1] A.P. Kater, et al., How does lenalidomide target the chronic lymphocytic leukemia microenvironment? Blood 124 (14) (2014) 21842189.

[2] J.A. Jones, J.C. Byrd, How will B-cell-receptortargeted therapies change future CLL therapy? Blood 123 (10) (2014) 1455-1460.

[3] Elisa ten Hacken and Jan A. Burger Molecular Pathways: Targeting the Microenvironment in Chronic Lymphocytic Leukemia-Focus on the BCell Receptor Clinical Cancer Research 20(3) February 1, 2014, 548-56 Published Online First December 9, 2013

[4] Han T-T, Fan L, Li J-Y, Xu W. Role of chemokines and their receptors in chronic lymphocytic leukemia: Function in microenvironment and targeted therapy. Cancer biology \& therapy. 2014;15:3-9.

[5] Burger JA. Chemokines and chemokine receptors in chronic lymphocytic leukemia (CLL): from understanding the basics towards therapeutic targeting. Semin Cancer Biol 2010; 20:424-30;

[6] Elisa ten Hacken, Jan A. Burger Microenvironment interactions and B-cell receptor signaling in Chronic Lymphocytic Leukemia: Implications for disease pathogenesis and treatment Biochimica et Biophysica Acta (BBA)- Molecular Cell Research Volume 1863, Issue 3 March 2016, Pages 401-413

[7] J.A. Burger, book S. Malek (ed.), Advances in Chronic Lymphocytic Leukemia, Advances in Experimental Medicine and Biology (2013) 792, Chapter 2 The CLL Cell Microenvironment

[8] Nicole S. Nicholas, Benedetta Apollonio, Alan G. Ramsay, Tumor microenvironment (TME)-driven immune suppression in B cell malignancy, Biochimica et Biophysica Acta 1863 (2016) 471482.

[9] Michele Dal Bo, Riccardo Bomben, Antonella Zucchetto, Giovanni Del Poeta, Gianluca Gaidano, Silvia Deaglio, Dimitar G. Efremov and Valter Gattei, Microenvironmental Interactions in Chronic Lymphocytic Leukemia: Hints for Pathogenesis and Identification of Targets for Rational Therapy, Current Pharmaceutical Design, 2012, 18, 33233334

[10] Caligaris-Cappio F Role of the microenvironment in chronic lymphocytic leukaemia. British Journal of Haematology, 2003, Nov, 123, 380-388.

[11] Jessie-F. Fecteau, Thomas J. Kipps, Structure and function of the hematopoietic cancer niche: focus on chronic lymphocytic leukemia Front Biosci(Schol Ed).(2004); 4: 61-73.

[12] Burger M, Hartmann T, Krome M, Rawluk J, Tamamura H, Fujii N, Kipps TJ, Burger JA. Small peptide inhibitors of the CXCR4 chemokine receptor (CD184) antagonize the activation, migration, and antiapoptotic responses of CXCL12 in chronic lymphocytic leukemia B cells. Blood 2005; 106:182430;

[13] Nicholas Chiorazzi and Manlio Ferrarini B cell chronic lymphocytic leukemia: Lessons Learned from Studies of the B Cell Antigen Receptor Annu. Rev. Immunol. 2003. 21:841-94

[14] Burger JA, Chiorazzi N. B cell receptor signaling in chronic lymphocytic leukemia. Trends Immunol. 2013;34(12):592-601.

[15] Burger JA, Burger M, Kipps TJ. Chronic lymphocytic leukemia B cells express functional CXCR4 chemokine receptors that mediate 
spontaneous migration beneath bone marrow stromal cells. Blood. 1999;94:3658-3667

[16] Alsagaby SA, Brennan P, Pepper C, Key Molecular Drivers of Chronic Lymphocytic Leukaemia (CLL), Clinical Lymphoma, Myeloma and Leukemia (2016), doi:10.1016/ j.clml.2016.08.008.

[17] Hoellenriegel J, Coffey GP, Sinha U, Pandey A, Sivina M, Ferrajoli A, Ravandi F, Wierda WG, O'Brien S, Keating MJ, et al. Selective, novel spleen tyrosine kinase (Syk) inhibitors suppress chronic lymphocytic leukemia B-cell activation and migration. Leukemia 2012; 26:1576-83;

[18] Ponader S, Chen SS, Buggy JJ, Balakrishnan K, Gandhi V, Wierda WG, Keating MJ, O'Brien S, Chiorazzi N, Burger JA. The Bruton tyrosine kinase inhibitor PCI-32765 thwarts chronic lymphocytic leukemia cell survival and tissue homing in vitro and in vivo. Blood 2012; 119:1182-9;

[19] Hoellenriegel J, Meadows SA, Sivina M, Wierda WG, Kantarjian H, Keating MJ, Giese N, O'Brien S, Yu A, Miller LL, et al. The phosphoinositide 3'-kinase delta inhibitor, CAL-101, inhibits B-cell receptor signaling and chemokine networks in chronic lymphocytic leukemia. Blood 2011; 118:360312;

[20] Garcia-Munoz R, Llorente L. Chronic lymphocytic leukemia: could immunological tolerance mechanisms be the origin of lymphoid neoplasms? Immunology 2014;142:536e40.

[21] Tadeusz Robak and Piotr Smolewski, Novel target to kill CLL Blood, January 2015 x Vol. 125, number 2

[22] Gobessi S, Laurenti L, Longo PG, Carsetti L, Berno V, Sica S, Leone G, Efremov DG. Inhibition of constitutive and BCR-induced Syk activation downregulates Mcl-1 and induces apoptosis in chronic lymphocytic leukemia B cells. Leukemia 2009; 23:686-97;

[23] Braselmann S, Taylor V, Zhao H, Wang S, Sylvain C, Baluom M, Qu K, Herlaar E, Lau A, Young C, et al. R406, an orally available spleen tyrosine kinase inhibitor blocks fc receptor signaling and reduces immune complex-mediated inflammation. J Pharmacol Exp Ther 2006; 319:9981008 ;

[24] Herman SE, Barr PM, McAuley EM, Delong Liu, Friedberg JW, Adrian Wiestner. Fostamatinib Inhibits BCR Signaling, and Reduces Tumor Cell Activation and Proliferation in Patients with Relapsed Refractory Chronic Lymphocytic Leukemia. 54th American society of hematology, 2012, 2882 Abstract

[25] J.A. Burger, A. Peled CXCR4 antagonists: targeting the microenvironment in leukemia and other cancers Leukemia, 23 (2009), pp. 43-52
[26] B. Stamatopoulos, N. Meuleman, C. De Bruyn, K. Pieters, P. Mineur, C. Le Roy, S. Saint-Georges, N. Varin-Blank, F. Cymbalista, D. Bron, L. Lagneaux AMD3100 disrupts the cross-talk between chronic lymphocytic leukemia cells and a mesenchymal stromal or nurse-like cell-based microenvironment: pre-clinical evidence for its association with chronic lymphocytic leukemia treatments Haematologica, 97 (2012), pp. 608-615

[27] Andritsos LA, Byrd JC, Hewes B, Kipps TJ, Johns D, Burger JA., Preliminary results from a phase I/II dose escalation study to determine the maximum tolerated dose of plerixafor in combination with rituximab in patients with relapsed chronic lymphocytic leukemia. Haematologica 2010; 95 Abstract 0772

[28] R. Berger, et al., Phase I safety and pharmacokinetic study of CT-011, a humanized antibody interacting with PD-1, in patients with advanced hematologic malignancies, Clin. Cancer Res. 14 (10) (2008) 3044-3051.

I. Sagiv-Barfi, et al.,Therapeutic antitumor immunity by check point blockade is enhanced by ibrutinib, an inhibitor of both BTK and ITK, Proc. Natl. Acad. Sci. U. S. A. 112 (9) (2015) E966-E972. [29] J. A. Dubovsky, et al., Ibrutinib is an irreversible molecular inhibitor of ITK driving a Th1-selective pressure in T lymphocytes, Blood 122 (15) (2013) 2539-2549.

[30] B.N. Lee, H. Gao, E.N. Cohen, X. Badoux, W.G. Wierda, Z. Estrov, S.H. Faderl, M.J. Keating, A. Ferrajoli, J.M. Reuben Treatment with lenalidomide modulates T-cell immunophenotype and cytokine production in patients with chronic lymphocytic leukemia Cancer, 117 (2011), pp. 39994008

[31] Vallet S, Palumbo A, Raje N, Boccadoro M, Anderson KC. Thalidomide and lenalidomide: Mechanism-based potential drug combinations. Leukemia \& Lymphoma (July 2008) 49(7): 1238 45.

[32] G. Aue, N. Njuguna, X. Tian, S. Soto, T. Hughes, B. Vire, K. Keyvanfar, F. Gibellini, J. Valdez, C. Boss, L. Samsel, J.P. McCoy Jr., W.H. Wilson, S. Pittaluga, A. Wiestner Lenalidomideinduced upregulation of CD80 on tumor cells correlates with T-cell activation, the rapid onset of a cytokine release syndrome and leukemic cell clearance in chronic lymphocytic leukemia Haematologica, 94 (2009), pp. 1266-1273

A. Acebes-Huerta, L. Huergo-Zapico, A.P. Gonzalez-Rodriguez, A. Fernandez-Guizan, A.R. Payer, A. Lopez-Soto, S. Gonzalez Lenalidomide induces immunomodulation in chronic lymphocytic leukemia and enhances antitumor immune responses mediated by NK and CD4 T cells BioMed. Res. Int., 2014 (2014), p. 265840 
[33] A.G. Ramsay, A.J. Johnson, A.M. Lee, G. Gorgun, R. Le Dieu, W. Blum, J.C. Byrd, J.G. Gribben Chronic lymphocytic leukemia $\mathrm{T}$ cells show impaired immunological synapse formation that can be reversed with an immunomodulating drug J. Clin. Invest., 118 (2008), pp. 2427-2437

[34] A.G. Ramsay, R. Evans, S. Kiaii, L. Svensson, N. Hogg, J.G. Gribben Chronic lymphocytic leukemia cells induce defective LFA-1-directed Tcell motility by altering Rho GTPase signaling that is reversible with lenalidomide Blood, 121 (2013), pp. 2704-2714

[35] R. Lapalombella, L. Andritsos, Q. Liu, S.E. May, R. Browning, L.V. Pham, K.A. Blum, W. Blum, A. Ramanunni, C.A. Raymond, L.L. Smith, A. Lehman, X. Mo, D. Jarjoura, C.S. Chen, R. Ford Jr., C. Rader, N. Muthusamy, A.J. Johnson, J.C. Byrd Lenalidomide treatment promotes CD154 expression on CLL cells and enhances production of antibodies by normal B cells through a PI3-kinasedependent pathway Blood, 115 (2010), pp. 26192629

[36] C. Galustian, B. Meyer, M.C. Labarthe, K. Dredge, D. Klaschka, J. Henry, S. Todryk, R. Chen, G. Muller, D. Stirling, P. Schafer, J.B. Bartlett, A.G. Dalgleish The anti-cancer agents lenalidomide and pomalidomide inhibit the proliferation and function of T regulatory cells Cancer Immunol. Immunother., 58 (2009), pp. 1033-1045

[37] Idler I, Giannopoulos K, Zenz T, Bhattacharya N, Nothing M, Dohner H, Stilgenbauer S, Mertens D. Lenalidomide treatment of chronic lymphocytic leukaemia patients reduces regulatory $\mathrm{T}$ cells and induces Th17 T helper cells. Br J Haematol. 2010; 148(6):948-50.

[38] R. Maffei, S. Fiorcari, J. Bulgarelli, L. Rizzotto, S. Martinelli, G.M. Rigolin, G. Debbia, I. Castelli, G. Bonacorsi, R. Santachiara, F. Forconi, D. Rossi, L. Laurenti, G.A. Palumbo, D. Vallisa, A. Cuneo, G. Gaidano, M. Luppi, R. Marasca Endotheliummediated survival of leukemic cells and angiogenesis-related factors are affected by lenalidomide treatment in chronic lymphocytic leukemia Exp. Hematol., 42 (2014), pp. 126-136 (e121)

[39] A.G. Ramsay,et al., Multiple inhibitory ligands induce impaired $\mathrm{T}$-cell immunologic synapse function in chronic lymphocytic leukemia that can be blocked with lenalidomide: establishing a reversible immune evasion mechanism in human cancer, Blood 120 (7) (2012) 1412-1421.

[40] R. Majeti, et al., CD47 is an adverse prognostic factor and therapeutic antibody target on human acute myeloid leukemia stem cells, Cell 138 (2) (2009) 286-299.

[41] M.P. Chao, et al., Anti-CD47 antibody synergizes with rituximab to promote phagocytosis and eradicate non-Hodgkin lymphoma, Cell 142 (5) (2010) 699-713.

[42] Tam CS, O'Brien S, Wierda W, et al. Longterm results of the fludarabine, cyclophosphamide, and rituximab regimen as initial therapy of chronic lymphocytic leukemia. Blood. 2008;112(4): 975980.

[43] Martijn HA van Attekum, Eric Eldering and Arnon P Kater Chronic lymphocytic leukemia cells are active participants in microenvironmental crosstalk Haematologica 2017 Volume 102(9):1469-1476 [44] Messmer D, Fecteau JF, O'Hayre M, Bharati IS, Handel TM, Kipps TJ. Chronic lymphocytic leukemia cells receive RAF-dependent survival signals in response to CXCL12 that are sensitive to inhibition by sorafenib. Blood 2011; 117:8829;

[45] McCaig AM, Cosimo E, Leach MT, Michie AM. Dasatinib inhibits B cell receptor signalling in chronic lymphocytic leukaemia but novel combination approaches are required to overcome additional prosurvival microenvironmental signals. Br J Haematol 2011; 153:199-211;

[46] Amrein PC, Attar EC, Takvorian T, Hochberg EP, Ballen KK, Leahy KM, Fisher DC, Lacasce AS, Jacobsen ED, Armand P, et al. Phase II study of dasatinib in relapsed or refractory chronic lymphocytic leukemia. Clin Cancer Res 2011; 17:2977-86.

[47] Quiroga MP, Balakrishnan K, Kurtova AV, et al. B-cell antigen receptor signaling enhances chronic lymphocytic leukemia cell migration and survival: specific targeting with a novel spleen tyrosine kinase inhibitor, R406. Blood 2009; 114 : 1029-37.

[48] Suljagic M, Longo PG, Bennardo S, et al. The Syk inhibitor fostamatinib disodium (R788) inhibits tumor growth in the Emu- TCL1 transgenic mouse model of CLL by blocking antigen-dependent Bcell receptor signaling. Blood 2010; 116: 4894-905

[49] Friedberg JW, Sharman J, Sweetenham J, et al. Inhibition of Syk with fostamatinib disodium has significant clinical activity in nonHodgkin lymphoma and chronic lymphocytic leukemia. Blood 2010; 115: 2578-85.

[50] Herman SE, Gordon AL, Wagner AJ, et al. Phosphatidylinositol 3kinase-delta inhibitor CAL101 shows promising preclinical activity in chronic lymphocytic leukemia by antagonizing intrinsic and extrinsic cellular survival signals. Blood 2010; 116 : 2078-88.

[51] Lannutti BJ, Meadows SA, Herman SE, et al. CAL-101, a p110delta selective phosphatidylinositol-3-kinase inhibitor for the treatment of B-cell malignancies, inhibits PI3K signaling and cellular viability. Blood 2011; 117: 591-4. 
[52] Liang X, Moseman EA, Farrar MA, et al. Tolllike receptor 9 signaling by CpG-B oligodeoxynucleotides induces an apoptotic pathway in human chronic lymphocytic leukemia B cells. Blood 2010; 115: 5041-52

[53] Castro JE, Prada CE, Loria O, et al. ZAP-70 is a novel conditional heat shock protein 90 (Hsp90) client: inhibition of Hsp90 leads to ZAP-70 degradation, apoptosis, and impaired signaling in chronic lymphocytic leukemia. Blood 2005; 106: 2506-12.

[54] van de Donk NW, Kamps S, Mutis T, Lokhorst HM. Monoclonal antibody-based therapy as a new treatment strategy in multiple myeloma. Leukemia 2011;

[55] Burger JA, Peled A. CXCR4 antagonists: targeting the microenvironment in leukemia and other cancers. Leukemia 2009; 23: 43-52.

[56] Kofler DM, Gawlik B, Elter T, GianellaBorradori A, Wendtner CM, Hallek M. Final Results of a Phase Ib Trial of Atacicept to Neutralize APRIL and BLyS in Patients with Refractory or Relapsed Chronic Lymphocytic Leukemia (CLL). Blood (ASH Annual Meeting Abstracts). 2009; 114:2373

[57] Campas C, Cosialls AM, Barragan M, IglesiasSerret D, Santidrian AF, Coll-Mulet L, de Frias M, Domingo A, Pons G, Gil J. Bcl-2 inhibitors induce apoptosis in chronic lymphocytic leukemia cells. Exp Hematol. 2006; 34(12):1663-9.

[58] O'Brien SM, Claxton DF, Crump M, Faderl S, Kipps T, Keating MJ, Viallet J, Cheson BD. Phase I study of obatoclax mesylate (GX15-070), a small molecule pan-Bcl-2 family antagonist, in patients with advanced chronic lymphocytic leukemia. Blood. 2009; 113(2):299-305.

[59] Tse C, Shoemaker AR, Adickes J, Anderson MG, Chen J, Jin S, Johnson EF, Marsh KC, Mitten MJ, Nimmer P, Roberts L, Tahir SK, Xiao Y, Yang $\mathrm{X}$, Zhang H, Fesik S, Rosenberg SH, Elmore SW. ABT-263: a potent and orally bioavailable $\mathrm{Bcl}-2$ family inhibitor. Cancer Res. 2008; 68(9): 3421-8.

[60] Vogler M, Furdas SD, Jung M, Kuwana T, Dyer MJ, Cohen GM. Diminished sensitivity of chronic lymphocytic leukemia cells to ABT-737 and ABT-263 due to albumin binding in blood. Clin Cancer Res. 2010; 16(16):4217-25.
[61] Wilson W, O'Connor OO, Roberts AW, Czuczman M, Brown J, Xiong H, Xiong H, Chiu Y, Krivoshik A, Enschede S, Humerickhouse R. ABT263 activity and safety in patients with relapsed or refractory lymphoid malignancies in particular chronic lymphocytic leukemia (CLL)/ small lymphocytic lymphoma (SLL). J Clin Oncol. 2009; 2009(27):8574.

[62] Zucchetto A, Benedetti D, Tripodo C, Bomben R, Dal Bo M, Marconi D, Bossi F, Lorenzon D, Degan M, Rossi FM. CD38/CD31, the CCL3 and CCL4 chemokines, and CD49d/vascular cell adhesion molecule-1 are interchained by sequential events sustaining chronic lymphocytic leukemia cell survival. Cancer research. 2009;69:4001-4009. .

[63] Burger JA, Quiroga MP, Hartmann E, et al. High-level expression of the T-cell chemokines CCL3 and CCL4 by chronic lymphocytic leukemia $\mathrm{B}$ cells in nurselike cell cocultures and after BCR stimulation. Blood 2009; 113: 3050-8.

[64] Sabroe I, Peck MJ, Van Keulen BJ, et al. A small molecule antagonist of chemokine receptors CCR1 and CCR3. Potent inhibition of eosinophil function and CCR3-mediated HIV-1 entry. J Biol Chem 2000; 275: 25985-92.

[65] Dau B, Holodniy M. Novel targets for antiretroviral therapy: clinical progress to date. Drugs 2009; 69: 31-50.

[66] Chang JT, Lichtenstein GR. Drug insight: antagonists of tumornecrosis factor-alpha in the treatment of inflammatory bowel disease. Nat Clin Pract Gastroenterol Hepatol 2006; 3: 220-8.

[67] Nesbitt A, Fossati G, Bergin M, et al. Mechanism of action of certolizumab pegol (CDP870): in vitro comparison with other antitumor necrosis factor alpha agents. Inflamm Bowel Dis 2007; 13: 1323-32.

[68] Melmed GY, Targan SR, Yasothan U, Hanicq D, Kirkpatrick P. Certolizumab pegol. Nat Rev Drug Discov 2008; 7: 641-2.

[69] Schmidt, S.M., Schag, K., Muller, M.R., Weck, M.M., Appel, S., Kanz, L., Grunebach, F. \& Brossart, P. Survivin is a shared tumor-associated antigen expressed in a broad variety of malignancies and recognized by specific cytotoxic T cells. Blood, $102,571-576$ 